\title{
Intersections
}

Canadian Journal of Music

Revue canadienne de musique

\section{Élizabeth Gallat-Morin. 2012. L'orgue de 1753 renaît de ses \\ cendres. Québec : Musée de la civilisation, 96 p. ISBN \\ 978-2-551-25342-5 (couverture souple)}

\section{Jean-Philippe Després}

Volume 34, numéro 1-2, 2014

URI : https://id.erudit.org/iderudit/1030877ar

DOI : https://doi.org/10.7202/1030877ar

Aller au sommaire du numéro

Éditeur(s)

Canadian University Music Society / Société de musique des universités canadiennes

ISSN

1911-0146 (imprimé)

1918-512X (numérique)

Découvrir la revue

Citer ce compte rendu

Després, J.-P. (2014). Compte rendu de [Élizabeth Gallat-Morin. 2012. L'orgue de

1753 renait de ses cendres. Québec : Musée de la civilisation, 96 p. ISBN

978-2-551-25342-5 (couverture souple)]. Intersections, 34(1-2), 190-194.

https://doi.org/10.7202/1030877ar

Copyright @ Canadian University Music Society / Société de musique des universités canadiennes, 2015
Ce document est protégé par la loi sur le droit d'auteur. L'utilisation des services d'Érudit (y compris la reproduction) est assujettie à sa politique d'utilisation que vous pouvez consulter en ligne.

https://apropos.erudit.org/fr/usagers/politique-dutilisation/ 
mot romantisme, au contraire, mais de montrer qu'il «participe toujours d'une construction esthétique ou idéologique, interprétant la musique et façonnant son histoire» (p. 373).

\section{RÉFÉRENCES}

Bénichou, Paul. 1973. Le Sacre de l'écrivain, 1750-1830. Essai sur l'avènement d'un pouvoir spirituel läque dans la France moderne. Paris : Librairie J. Corti.

Dahlhaus, Carl. 2013. Fondements de l'histoire de la musique, traduit de l'allemand par Marie-Hélène Benoît-Otis. Arles : Actes Sud/Cité de la musique.

___.1997. L'idée de la musique absolue : une esthétique de la musique romantique, traduit de l'allemand par Martin Kaltenecker. Genève: Contrechamps. Einstein, Alfred. 1959. Music in the Romantic Era. New York: W.W. Norton.

Hoffmann, E.T.A. 1985 [1810]. «Ludwig van Beethoven, Cinquième Symphonie (Avril-Mai 1810)», Écrits sur la musique, traduit de l'allemand par Brigitte Hébert et Alain Montandon, 38-51. Lausanne : L'Âge d'homme.

Plantinga, Leon. 1989. La musique romantique. Histoire du style musical au $X I X^{e}$ siècle en Europe, traduit de l'américain par Dennis Collins. Paris : J.C. Lattès.

Rosen, Charles. 2002 [1995]. La génération romantique: Chopin, Schumann, Liszt et leurs contemporains, traduit de l'anglais par Georges Bloch. Paris : Gallimard.

_. 1993. Plaisir de jouer, plaisir de penser : conversation avec Catherine Temerson. Paris : Eshel, Genève : Georg.

Treitler, Leo. 1989. Music and the Historical Imagination. Cambridge : Harvard University Press.

Bruno Coulombe

Élizabeth Gallat-Morin. 2012. L'orgue de 1753 renaît de ses cendres. Québec : Musée de la civilisation, 96 p. ISBN 978-2-551-25342-5 (couverture souple).

Tous les instruments musicaux fabriqués de main d'homme, que ce soit par un facteur ou par un luthier sont, par définition, uniques. De ceux-ci, l'orgue est probablement l'instrument dont les traits distinctifs sont les plus marqués d'un modèle à l'autre. En effet, les éléments constitutifs de l'orgue, par exemple la forme de son buffet, le nombre de ses claviers ou encore le choix des jeux qui le composent, sont propres à chaque instrument.

Dans le même ordre d'idées, chaque orgue est chargé d'une histoire elle aussi unique, tributaire des circonstances particulières liées à sa commande, à sa fabrication, à son installation et à son utilisation à une époque et dans un lieu donnés. Élisabeth Gallat-Morin nous livre l'un de ces récits dans son ouvrage intitulé L'orgue de 1753 renaît de ses cendres, qui se révèle, au fil des pages, être une véritable épopée. 
Lauteure expose d'abord le contexte historique entourant la commande, la fabrication et l'installation de «l'orgue de 1753" puis le parcours déterminé, passionné et rigoureux qui a mené à sa reconstruction, 250 ans après sa destruction lors du siège de Québec de 1759. À l'instigation du renommé claveciniste et organiste canadien Kenneth Gilbert, la restauration de cet instrument n’a pu se concrétiser que grâce aux efforts conjugués de musicologues, de facteurs d'instruments et de musiciens passionnés, ainsi qu'à l'aide et au soutien de nombreux donateurs et du Musée de la civilisation de Québec.

Outre son apport scientifique aux domaines de la musicologie et de l'organologie, cet immense chantier de recherche offre, depuis 2009, la chance au grand public de découvrir et d'entendre un instrument unique aux sublimes sonorités d'époque.

Dès le premier coup d'œil, le livre d'Élisabeth Gallat-Morin se démarque par le grand soin apporté à sa conception graphique. En effet, la clarté de sa mise en page accorde au texte toute la place qu'il mérite. Par ailleurs, cette riche source d'informations ne saurait être mieux supportée sur le plan visuel qu'elle ne l'est. En effet, de superbes illustrations parsèment l'ouvrage, lesquelles consistent principalement en des reproductions de documents d'époque et des photos entourant la construction et l'installation au Musée de l'Amérique française de l'orgue Juget-Sinclair, Opus 35.

L'ouvrage se divise en trois grandes sections. La première, «L'histoire des orgues et de la vie musicale dans la paroisse de Québec», brosse un portrait chronologique des évènements qui ont précédé la commande de l'orgue de 1753. Ce préambule couvre une période relativement longue, s'étalant de 1633 à 1753 . Il trace notamment l'évolution du lieu de culte principal dans la ville de Québec - qui, en 1633, était situé à l'église Notre-Dame-de-la-Recouvrance, en haute ville, et qui a ensuite été relocalisé à l'emplacement de la future cathédrale Notre-Dame de Québec, dans le vieux Québec ${ }^{5}$ - ainsi que les trois orgues auxquelles succède l'orgue de 1753. Ainsi, la première section du livre de GallatMorin offre une vue d'ensemble du lien entre le social, le religieux et la musique en Nouvelle-France à cette époque qui précède la conquête britannique, en posant les fondements historiques nécessaires à une compréhension éclairée de la section suivante qui se rapporte spécifiquement à la commande de l'orgue de 1753. Plus précisément, le lecteur y découvrira comment l'érection en diocèse de la Nouvelle-France, couplée à la croissance démographique de la région, entraîne, dans un premier temps, la construction d'une nouvelle cathédrale à Québec (achevée en 1697), puis de substantiels travaux d'agrandissement de cette même église, une cinquantaine d'années plus tard. Cette nouvelle église, dont les dimensions ont plus que doublées, nécessite l'achat d'un orgue plus puissant en termes de projection sonore.

La deuxième section, intitulée "L'orgue Richard», relate l'ensemble des informations historiques accessibles concernant l'orgue de 1753, de sa commande

5 Ravagée par des incendies (en 1759 et 1922) la cathédrale est reconstruite à deux reprises en son emplacement d'origine. 
par le chanoine Jean-Marie de La Corne de Chaptes ${ }^{6}$, jusqu'à sa destruction en 1759. Entre ces deux moments charnières, l'auteure discute également des aspects liés à la construction de l'instrument, à son transport, à son installation, ainsi qu'à sa trop brève utilisation. Nous pourrons notamment apprendre, dans cette partie de l'ouvrage, qu'en dépit des responsabilités qui lui incombèrent à l'époque, les connaissances en matière d'orgues du chanoine La Corne étaient bien limitées. Il eût donc à fureter informations et conseils çà et là, afin de s'acquitter de ses obligations et de passer une commande éclairée au facteur parisien Robert Richard7.

La section finale du livre, intitulée "L'orgue de 1753 renaît de ses cendres", détaille toutes les étapes entourant la reconstitution de l'orgue Richard, de l'émergence du projet de sa reconstruction, jusqu'aux concerts qui ont été donnés, depuis son inauguration, sur l'instrument achevé. L'idée de redonner vie à cet instrument est née en 1998 chez le musicien québécois Kenneth Gilbert, qui signe l'introduction de la dernière section du livre de Gallat-Morin. Afin de concrétiser son projet, Kenneth Gilbert a constitué, la même année, le Comité pour la reconstruction de l'orgue Richard 1753 qui a établi, dès sa première réunion, le Musée de l'Amérique française (c'est-à-dire l'ancienne chapelle extérieure du Séminaire) comme étant l'endroit tout désigné pour recevoir l'orgue. Il est intéressant de noter que le musée se situe à 50 mètres de l'emplacement d'origine de l'orgue de 1753. Pour donner suite à cette introduction, l'auteure décrit les efforts déployés par le Comité pour la reconstruction de l'orgue Richard 1753-dont elle fait elle-même partie - et par les différents contributeurs au projet. Tout le travail qui a été accompli a finalement été récompensé par la reconstitution de l'orgue en 2009. À ce bref récit succède une contribution très intéressante, rédigée par le facteur Denis Juget, de l'entreprise Juget-Sinclair de Montréal, sur laquelle le comité a jeté son dévolu pour mener à bien ce projet. Lorganier y décrit à la fois les attributs de l'instrument qu'il a fabriqué et le processus décisionnel qui a permis de les déterminer. À cette dernière contribution succède celle d'Hélène Dionne, éditrice du Musée de la civilisation, qui décrit les travaux d'aménagements qui ont dû être effectués dans la Chapelle de l'Amérique française afin de pouvoir procéder à une installation sécuritaire du nouvel orgue. Finalement, l'auteure principale reprend le flambeau pour clore l'ouvrage en présentant la liste des évènement lors desquels l'orgue a pu être entendu, depuis son inauguration officielle le 4 octobre 2009 par Michel Bouvard, organiste de la Basilique Saint-Sernin de Toulouse et professeur d'orgue au Conservatoire National Supérieur de Paris.

6 Le chanoine Jean-Marie de La Corne de Chaptes (1714-1779), nommé chanoine en 1747, est mandaté en 1750 par le Chapitre de Québec pour défendre ses intérêts en France dans un procès intenté contre le Séminaire de Québec. D’abord retenu en Europe par les affaires du Chapitre, puis par la conquête britannique, son séjour en Europe, qu'il espérait bref, se prolongea finalement jusqu'à sa mort.

7 Robert Richard "parait s'être adonné plus aux petits instruments mécaniques, qu'aux grandes orgues» (Pierre 1893, p. 96). Fort inventif, il «a obtenu un atelier à la bibliothèque du Roi » (Tableau de Paris 1759, p. 212) où il crée, en plus de ses orgues et serinettes, des automates et diverses inventions (diapason et métronome) liées à la musique. 
Pierre angulaire de l'ouvrage, l'orgue Juget-Sinclair, Opus 35 est une reconstitution d'un instrument de facture classique française du $18^{\mathrm{e}}$ siècle. L'entreprise Juget-Sinclair ne disposait que de bien peu d'informations pour s'assurer de la fidélité historique de leur reproduction. En effet, aucune illustration ou aucun plan de l'orgue original n'ont été retrouvés à ce jour. Cependant, en plus de l'acte notarié qui décrivait les jeux que devait comporter l'instrument, les organiers ont pu trouver de précieux indices dans une lettre adressée par le chanoine La Corne au Chapitre de Québec :

« [...] Celui que je vous envoie en serrait [sic] le Positif sans y rien ajouter ni changer et nous aurions un orgue semblable à ceux de Saint-Eustache, Saint-Médéric, les Petits-Pères, qui sont les plus beaux de Paris [...] » (cité par Gallat-Morin 2009, p. 34).

Les trois instruments auxquels réfère le Chanoine dans sa lettre étant de grands orgues de tribune avec positifs de dos, les facteurs ont déduit que l'orgue de 1753 devait également respecter ce type de configuration. À partir de ces sources et de leur expertise historique, les facteurs ont construit un magnifique orgue à traction mécanique - le seul de la sorte se retrouvant à l'intérieur des murs de la Vieille-Capitale - à un clavier et un pédalier, comportant 10 jeux. L'histoire nous apprend que le jeu de tierce, typique de la facture française de l'époque (Owen, N.A.), a été ajouté à la hâte au contrat notarié, sous les conseils de Jean Landrin, un des organistes œuvrant à la cour de Louis XV. Deux soufflets fournissent le vent nécessaire au bon fonctionnement de l'instrument, ces derniers peuvent soit être actionnés par un souffleur ou - grâce à un ajout anachronique essentiel - par ventilation électrique. Une autre caractéristique intéressante de l'orgue Juget-Sinclair, Opus 35 est l'accord de l'instrument, qui, comme il était d'usage à l'époque en France, est ajusté sur un diapason relativement grave (à $392 \mathrm{~Hz}$, donc un ton sous le la $44 \mathrm{OHz}$ ), suivant le tempérament mésotonique pur. Toutes les quintes du tempérament mésotonique pur, hormis une sont légèrement abaissées. L'exception se trouve entre sol dièse et mi bémol, cet intervalle, appelé «quinte du loup» se trouve ainsi à être substantiellement haussé, ce qui a pour effet de rendre certaines tonalités pratiquement inutilisables (Kent 1999, 44).

En raison du peu de détails pouvant être puisés des sources historiques au sujet de l'orgue de 1753, un certain nombre de décisions ont dues être prises par l'entreprise Juget-Sinclair lors de la conception et la construction de leur Opus 35. À titre d'exemple, ils ont dû établir l'endroit de coupure des jeux sur le clavier ainsi que la forme du buffet. Ces nombreuses inconnues font en sorte que, sauf improbable coïncidence, la réplique ne saurait être une copie conforme de l'orgue original. Ceci, à mon humble avis, plutôt que d'affaiblir la démarche des facteurs et du Comité pour la reconstruction de l'orgue Richard 1753, renforce la valeur et la nécessité de leur travail. Ainsi, l'orgue Juget-Sinclair, Opus 35, plutôt que de n'être qu'une imitation de l'original, se trouve bonifié par la conjugaison des savoir-faire d'autrefois et d'aujourd'hui.

Avant de terminer, de petits bémols sont à apporter sur le livre de GallatMorin. D'abord, dans les deux premières parties de l'ouvrage, le fil conducteur 
entre les différents thèmes abordés est le plus souvent ténu. De plus, certains sujets ne sont pas développés, une fois amenés. Ces faiblesses sont évidemment causées par la quantité limitée de sources historiques disponibles. Cependant, à mon avis, les transitions entre les différents aspects traités auraient pu être rendues plus fluides par l'auteure afin d'adoucir cette impression d'énumération chronologique d'évènements qui est susceptible de surprendre ou même de distraire le lecteur par endroits. Par la suite, sans vouloir réduire l'importance des contributions respectives des multiples acteurs qui ont rendu possible la réalisation de ce projet de restauration, l'ouvrage prend parfois les allures d'une lettre de remerciements. Une bonne partie de ces mots de reconnaissance aurait eu avantage à être déplacée vers les pages liminaires du livre, afin d'accorder plus d'importance, dans les pages centrales, aux informations se rapportant à l'orgue à proprement parler, qui revêtent un plus grand intérêt pour le lecteur. Finalement, en raison de ma passion pour la facture instrumentale, l'excellente section traitant de la construction et des caractéristiques de l'orgue reconstitué, rédigée par le facteur d'orgue Denis Juget, m'est apparue trop brève, surtout en regard de la valeur et de la pertinence des questions qui y sont soulevées. J'aurais aimé en savoir davantage à propos des différentes étapes du travail de ces facteurs ainsi que sur les choix qu'ils ont dû effectuer tout au long du processus de reconstruction de l'orgue Richard. Ceci étant dit, L'orgue de 1753 renaît de ses cendres est un ouvrage magnifique et fort bien documenté, un incontournable pour les passionnés de l'orgue et de la vie musicale en Nouvelle-France.

\section{RÉFÉRENCES}

Kent, Christopher. 1999. "Temperament and pitch». The Cambridge Companion to the Organ, $1^{\mathrm{er}}$ édition, 42-54. Cambridge : Cambridge University Press. Cambridge Companions Online. http://dx.doi.org.ezproxy.bibl .ulaval.ca/10.1017/CCOL9780521573092.005 (Page consultée le 6 janvier 2014).

Pierre, Constant. 1893. Les facteurs d'instruments de musique, les luthiers et la facture instrumentale. Paris : Sagot. http://archive.org/stream/ lesfacteursdinso1piergoog\#page/n115/mode/1up (Page consultée le 25 mars 2014).

Pelletier, Jean-Guy. 2003. "La corne de Chaptes, Joseph-Marie de». Dictionnaire biographique du Canada, vol. 4, Québec: Université Laval/University of Toronto. http://www.biographi.ca/fr/bio/la_corne_de_chaptes_joseph_marie_de_4F.html (Page consultée le 24 mars 2014)

Owen, Barbara et Peter Williams. «The French classical organ». Grove Music Online. Oxford Music Online.http://www.oxfordmusiconline.com.ezproxy .bibl.ulaval.ca/subscriber/article/grove/music/4401opg5 (Page consultée le 6 janvier 2014).

Tableau de Paris. 1759. Cité par Constant Pierre, Les facteurs d'instruments de musique, les luthiers et la facture instrumentale. Paris : Sagot, 1893. http:// archive.org/stream/lesfacteursdinso1piergoog\#page/n115/mode/1up (Page consultée le 25 mars 2014). 